\title{
Segmentation of Vascular Structures and Hematopoietic Cells in 3-D Microscopy Images and Quantitative Analysis
}

\author{
Jian $\mathrm{Mu}^{a}$, Lin Yang ${ }^{a}$, Malgorzata M. Kamocka ${ }^{b}$, \\ Amy L. Zollman ${ }^{b}$, Nadia Carlesso ${ }^{b}$ and Danny Z. Chen ${ }^{a}$ \\ ${ }^{a}$ Department of Computer Science and Engineering, University of Notre Dame, \\ Notre Dame, Indiana 46556, USA \\ ${ }^{b}$ Department of Pediatrics, Indiana University School of Medicine, \\ Indianapolis, Indiana 46202, USA
}

\begin{abstract}
In this paper, we present image processing methods for quantitative study of how the bone marrow microenvironment changes (characterized by altered vascular structure and hematopoietic cell distribution) caused by diseases or various factors. We develop algorithms that automatically segment vascular structures and hematopoietic cells in 3-D microscopy images, perform quantitative analysis of the properties of the segmented vascular structures and cells, and examine how such properties change. In processing images, we apply local thresholding to segment vessels, and add post-processing steps to deal with imaging artifacts. We propose an improved watershed algorithm that relies on both intensity and shape information and can separate multiple overlapping cells better than common watershed methods. We then quantitatively compute various features of the vascular structures and hematopoietic cells, such as the branches and sizes of vessels and the distribution of cells. In analyzing vascular properties, we provide algorithms for pruning fake vessel segments and branches based on vessel skeletons. Our algorithms can segment vascular structures and hematopoietic cells with good quality. We use our methods to quantitatively examine the changes in the bone marrow microenvironment caused by the deletion of Notch pathway. Our quantitative analysis reveals property changes in samples with deleted Notch pathway. Our tool is useful for biologists to quantitatively measure changes in the bone marrow microenvironment, for developing possible therapeutic strategies to help the bone marrow microenvironment recovery.
\end{abstract}

Keywords: 3-D microscopy images, vascular structure segmentation, cell segmentation, quantitative analysis

\section{INTRODUCTION}

The bone marrow is the site of hematopoietic development, and is the origin of multiple diseases, including disorder of Notch signaling pathway, leukemia, myeloma, and myelodysplastic syndrome. Such diseases may trigger changes in the bone marrow microenvironment, and the changes are often characterized by altered vascular structures and spatial organization of hematopoietic cells surrounding the vessels. ${ }^{1-3}$ The changes in the bone marrow microenvironment may also be caused by factors such as exposure to radiation or receiving chemotherapy-induced hemato-toxicity. ${ }^{4}$ Recent advances in medical imaging techniques have allowed for highresolution and real-time imaging of the bone marrow. However, a significant problem limiting the use of imaging techniques and their applications to broader questions is the lack of quantitative analytical and statistical tools, especially for 3-D and 4-D images.

In this study, we develop new methods to quantify properties of vascular structures and hematopoietic cells in 3-D microscopy images, and quantitatively study how these properties change with factors that trigger changes in the bone marrow microenvironment. Studying how these factors affect the bone marrow microenvironment may help deepen the understanding of the mechanisms of different components in the bone marrow microenvironment. In addition, our tool can assist biologists and physicians for various research and clinical purposes. For example, it can be used to screen drug hemato-toxicity and to determine the efficacy of drugs or mitigators to protect the hematopoietic system and the bone marrow microenvironment. It can also be used as a tool for discovery to gain insights in the relation between the bone marrow microenvironment and hematopoietic cells during hematopoietic/vascular regeneration, leukemia development, recovery and relapse.

Corresponding author: Jian Mu. E-mail: jmu@nd.edu. Telephone: +1 (574) 631-8804.

Medical Imaging 2015: Image Processing, edited by Sébastien Ourselin, Martin A. Styner, Proc. of SPIE Vol. 9413 , $941305 \cdot$ C $2015 \mathrm{SPIE} \cdot \mathrm{CCC}$ code: $1605-7422 / 15 / \$ 18 \cdot$ doi: $10.1117 / 12.2082350$

This is the author's manuscript of the article published in final edited form as:

Mu, J., Yang, L., Kamocka, M. M., Zollman, A. L., Carlesso, N., \& Chen, D. Z. (2015). Segmentation of vascular structures and hematopoietic cells in 3D microscopy images and quantitative analysis. In SPIE Medical Imaging (Vol. 9413, pp. 941305-941305-10). International Society for Optics and Photonics. http://doi.org/10.1117/12.2082350 


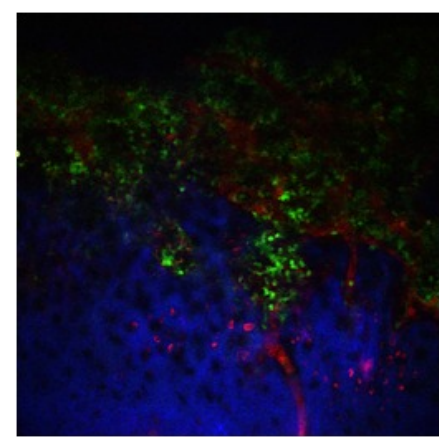

(a)

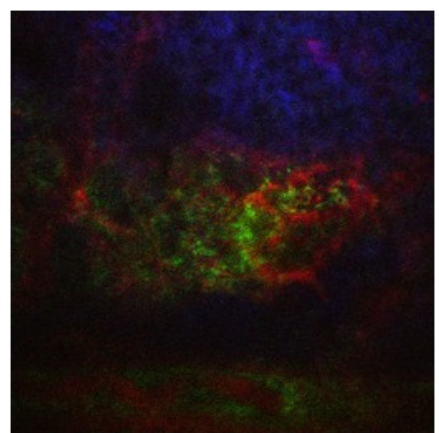

(b)

Figure 1. Sample images: (a) a 2-D slice from a controlled type stack; (b) a 2-D slice from a knock-out type stack (viewed better in color)

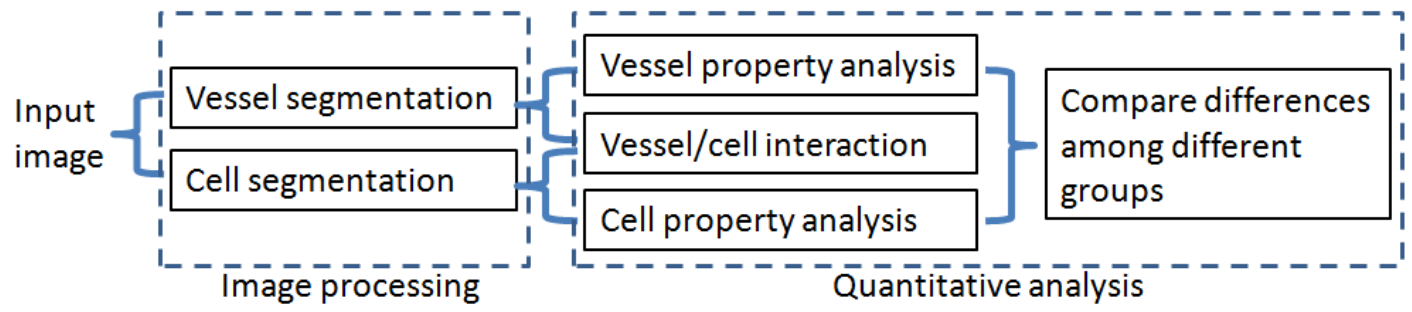

Figure 2. Flowchart of our proposed method.

In our experiment, we delete a component of Notch signaling pathway in mice. These mice then develop a form of leukemia, with observable changes in the bone marrow over time. These samples are called the knock-out type, and imaged using 3-D 2-photon microscopy. The deletion of Notch signaling pathway triggered observable changes in the vascular structures and distribution of hematopoietic cells in the bone marrow. We compare the knock-out type with the normal samples (called the controlled type) to quantitatively examine how the properties of vascular structures and hematopoietic cells change in the knock-out type with respect to the controlled type samples.

Each 3 -D image is a stack of 2 -D slices. The size of a typical image is $512 \times 512 \times 90$. The interval between consecutive slices in the actual samples is $1 \mu \mathrm{m}$. Fig. 1(a) and Fig. 1(b) show one 2-D slice from a controlled type and a knock-out type image, respectively. In the images, red is for vascular structures, green is for hematopoietic cells, and blue is for the rest of the bone marrow components, collagen and bone.

To quantitatively analyze properties of vascular structures and hematopoietic cells, we need to first segment them in the images. The segmentation problem is quite challenging due to various imaging artifacts existing in the images: the images are often very fuzzy; the image intensity usually varies significantly from slice to slice, and even within the same slice. The vessel surfaces shown in the images are not smooth, with lots of bumps and cavities, partly due to some vessel voxels not receiving sufficient fluorescent signal, or some blood cells that are attached to the vessel wall and exhibit the same color signal as vessels. The hematopoietic cells are also fuzzy. Some cells lump together with no clear separation among one another.

We segment the vessels using local thresholding, with post-processing steps to deal with imaging artifacts. Our cell segmentation algorithm is an improved watershed algorithm that relies on both intensity and shape information, and can segment multiple overlapped cells better than common watershed methods. We quantitatively compute various features of vascular structures and cells. In analyzing vascular properties, we give algorithms for pruning fake vessel segments and branches. Our analysis results reveal structural changes of vascular structures and cell distribution in the knock-out type samples. The research presented in this paper is our original work which is not under consideration elsewhere. 


\section{RELATED WORK}

Many approaches have been designed to segment vascular structures from images. The most widely-studied vascular networks include retinal vascular networks ${ }^{5-11}$ and cerebral vascular networks ${ }^{12,13}$ in CT or MR images. The vessels are usually segmented using methods such as Laplacian operators, ${ }^{5}$ watershed algorithm, ${ }^{7}$ line masks, ${ }^{8,13}$ supervised classifiers ${ }^{6,9,10}$ based on vessel features (e.g., tortuosity, neighborhood context, etc.), or interactive methods with user intervention. ${ }^{11,12}$

Different types of vascular images usually vary greatly in the visual properties and imaging artifacts, as well as the challenges posed to the segmentation of vessels. For example, the main challenge posed by retinal images is that the contrast between vessels and background is quite low; where as in cerebral images, vessels are usually quite small. Algorithms for these images are usually specifically designed based on the features of the images and target objects in the images. Therefore, algorithms designed for other types of images usually do not work well on other types of images.

The image analysis software suite ImageJ has a vascular network toolkit for segmenting vessels in 3-D images. However, it requires that the contrast between vessels and background be high, and image intensity be homogeneous throughout all slices. In addition, it requires all vessels to be of regular tubular shape and of roughly the same thickness. With imaging artifacts that we face, ImageJ cannot segment vascular structures in our images. However, to our best knowledge, no known algorithm was specifically designed to automatically segment vessels in the bone marrow from 3-D microscopy images.

Many cell segmentation algorithms rely on multiple cell features, such as membrane and nuclei, ${ }^{14,15}$ which are not shown in our images. The watershed method is commonly used to deal with this type of images, but it does not handle multiple overlapping cells sufficiently well.

\section{METHODOLOGY}

Fig. 1 shows the flowchart of our proposed method, consisting of two stages. In the first stage, we segment the vessels and cells in images. In the second stage, we quantitatively compute properties of the segmented vascular structures and cells, and compare the differences in the properties between images of different types.

\subsection{Segmentation of Vascular Structures}

In our images, vessels usually do not exhibit specific shape patterns. Therefore, methods based on vessel shape features (i.e., straight lines or curves with specific curvatures) ${ }^{8,9,13}$ usually would not work well on our images. Since the vessel surfaces could sometimes be very fuzzy, approaches based on edge features may also fail. ${ }^{5}$ The intensity of vessel voxels is usually higher than non-vessel voxels in their neighborhoods. Therefore, we segment vessels by thresholding.

The main idea for segmenting vascular structures from images is to first extract vessel volumes by thresholding, then add post-processing steps to refine the segmentation. The algorithm consists of the following steps: (1) vessel thresholding; (2) cavity filling and (3) surface smoothing.

1. Vessel thresholding. Vessels are colored by red dextran and exhibit high red signals in the images. Thus, vessel volumes can be extracted from the images by setting a proper cut-off value for the red channel. We first segment vessels by computing the threshold locally using Otsu's method. The threshold value for each voxel is determined by the voxels in its close neighborhood, with the neighborhood radius being the approximate size of the thickest vessel in the corresponding type of images. This neighborhood radius ensures that most neighborhood circles contain sufficient voxels for both vessel and non-vessel; thus a proper threshold value can be chosen. For the same type of images, the sizes of the thickest vessels are close. Therefore, we use the same neighborhood radius for all images of the same type. The thresholds are not allowed to be smaller than 10 (out of 255), i.e., if a computed threshold value is below 10, we use 10 as the threshold instead. Fig. 3(b) shows the thresholded image from Fig. 3(a).

2. Cavity filling. The results generated by directly applying thresholding techniques are usually not satisfactory enough. For example, although local thresholds are used to extract the vessels, there is no guarantee that all vessel voxels be extracted, and all background voxels be excluded. There are cells and other components 


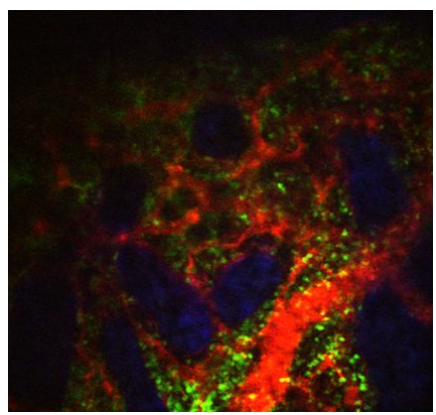

(a)

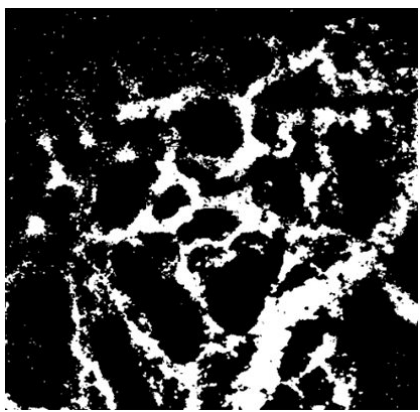

(b)

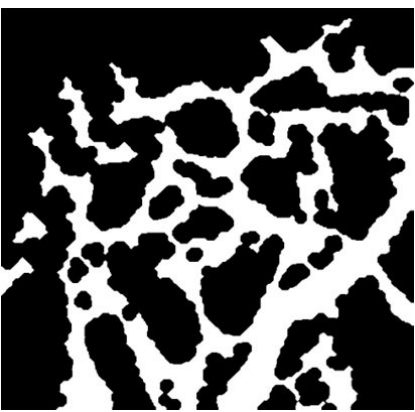

(c)

Figure 3. Vessel segmentation: (a) A 2-D slice in a 3-D image; (b) thresholded image; (c) the image after post-processing.

inside vessels that do not absorb red dextran, and thus appear as dark points in the images. These close cavities cannot exist in real vascular structures, and may cause problems in the analysis of vessels. For example, for the vessel skeletonization algorithm in Section 3.3, close cavities inside vessels will result in close chambers in the vessel skeletons, which do not correspond to any real structures in the vascular networks.

We find and fill all close cavities inside the vessels based on the flood fill method. A seed point is picked from space outside of the vascular structures (e.g., above the top slice of the 3-D image). Region growing is performed from the seed point to include all background voxels reachable from the seed point. Background voxels not reachable from the seed point are considered as cavities.

3. Surface smoothing. Another problem is that the segmented vessel surfaces usually do not look smooth. There are particles of red dextran that leak outside of vessels and are incorporated with bone cells in the marrow. These red-colored bone cells, along with the scattered red dextran particles, have the same color signal as vessels. Their intensity may even be very high. Thresholding will identify these cells and points as the same type of object as vessels. Therefore, the segmented vessel surfaces using thresholding are usually not smooth due to the existence of these unrelated components. They should all be excluded from the segmentation results.

We apply morphological operations to smooth the vessel surfaces, and remove small unrelated components. These small components to be removed include red blood cells, isolated red voxels caused by diffusion of red dextran, and other fuzzy areas in which no clear structure can be seen. Based on our experiments, disk of radius 3 for an open operation followed by a close operation can effectively remove these components without affecting the major vascular structures in our images. Fig. 3(c) shows the vessels after surface smoothing. Note that the "holes" in Fig. 3(c) are just vessel loops; all close cavities in 3-D have been filled in this example.

\subsection{Segmentation of Hematopoietic Cells}

Since many cell components, such as cell nuclei and membrane, are not visible in our images, cell segmentation algorithms based on such cell features ${ }^{14,15}$ would not work on our images. In our images, hematopoietic cells have higher green value than neighboring regions, and most cells have higher intensity in the middle than near the boundary. Based on these features, we design an improved watershed algorithm to segment the cells.

Similar to segmenting vascular structures, hematopoietic cells are also segmented first by local thresholding. Due to the existence of large cell regions, to ensure that each neighborhood circle contains sufficient voxels for both cell and non-cell, we choose a neighborhood radius of 100 voxels. We then apply the open operation to remove scattered green points extracted by thresholding.

Applying the watershed algorithm on distance-transformed images can separate partially-overlapped round objects. However, it usually does not separate target objects quite well if the target objects are not of round shapes, or there are multiple objects lumping together.

We observe that most cells have higher intensity in the middle than near the boundary. The top image in Fig. 4(a) show intensity of two touching cells. The height of the curve corresponds to the intensity values. This feature is similar to that of the distance-transformed images (the middle image in Fig. 4(a)) in that they 


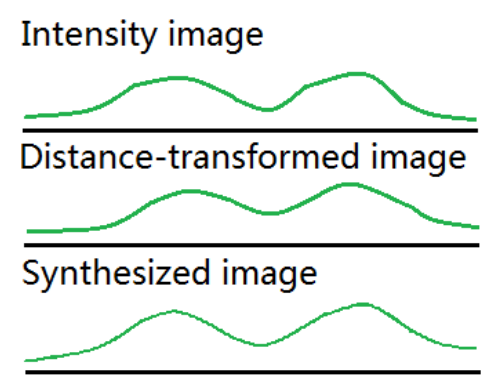

(a)

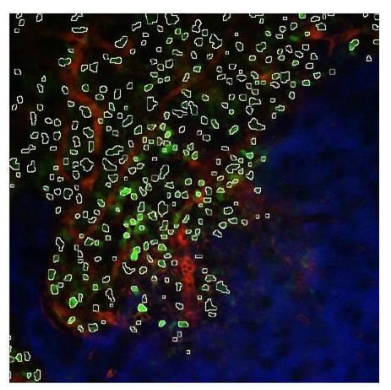

(b)

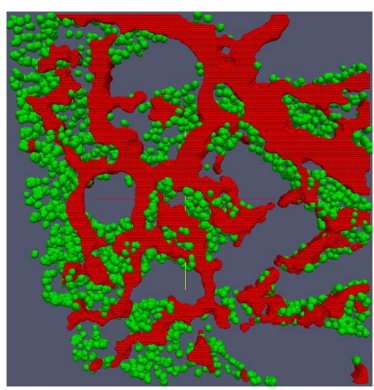

(c)

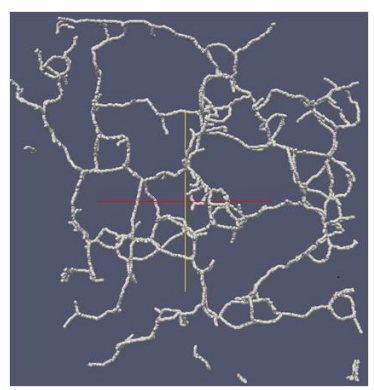

(d)

Figure 4. Cell segmentation and vessel skeletonization: (a) Overlapping intensity and distance-transformed images for segmentation; (b) a 2-D slice showing segmented cells (viewed better in color); (c) visualizing segmented vessels and cells in 3-D; (d) skeleton of vascular network

both have high intensities in the middle of cells and relatively low intensities on the cell boundaries and in the conjunction parts. Based on this observation, we design an improved watershed method to segment the cells by overlapping these two types of images using a weighted sum. First, distance transformation is performed on the thresholded images. Each connected component in the thresholded image is viewed as a point set $S$. We find $S^{\prime} \subseteq S$ such that each point in $S^{\prime}$ has at least one 4-connected neighboring point not in $S$. For each pixel $p \in S$, its intensity is re-computed using the formula

$$
I_{D T}(p)=a \cdot \min _{q \in S^{\prime}} \operatorname{dist}(p, q)+b \cdot I(p),
$$

where $\min _{q \in S^{\prime}} \operatorname{dist}(p, q)$ is the Euclidean distance from $p$ to the nearest point on the boundary of the connected component that $p$ belongs to. This distance is normalized to $[0,255]$ for all points in $S$. $I(p)$ is the green channel value (of range $[0,255]$ ) of $p$ in the original image. $a$ and $b$ are coefficients of the two terms. Based on our experiments, $a$ and $b$ are both set to 0.5 for best segmentation quality. The bottom image in Fig. 4(a) is overlaid using the intensity and distance-transformed images. Watershed segmentation is then performed on this synthesized images.

This improved watershed segmentation is more robust than relying only on a single feature because if one feature fails to capture cells at some locations, it usually can be offset by other features. For example, if the cell conjunction part is flat in either the intensity or distance-transformed images due to imaging artifacts, while still concave in the other, the it will still be concave in the synthesized image, and watershed algorithm can still identify the cell conjunction parts to separate touching cells. Fig. 4(b) shows the segmented hematopoietic cells in one image slice. Fig. 4(c) shows the segmented vascular structure and hematopoietic cells in 3-D.

\subsection{Analysis Results}

After the vessels and cells are segmented, we quantitatively compute a set of their properties and examine the differences between samples of the two types. We analyze the properties of vessels and cells independently, and then study the interactions between vessels and cells.

\subsubsection{Vessel skeletonization}

The segmented vessel volumes (red components in Fig. 4(c)) contain only vessels and exclude all other components in the marrow. However, vessels in the segmented images are of various shapes, which make the computation of many properties difficult. We simplify their structures by computing the skeleton of the vascular network. The skeleton is a one-voxel wide succinct representation of the vascular network which retains the geometric and topological properties of vessels, such as connectivity, branching, topology, length, and direction. It can be computed by repeatedly peeling off points on the boundary of the object, until only single lines are left. ${ }^{16}$ The skeletonization is an iterative process. In each iteration, the skeletonization algorithm checks every voxel in the image to determine if it is removable, and remove all voxels that can be removed. This process is repeated until no voxels in the image can be removed. A removable voxel must satisfy the following conditions: 
(a) The voxel is on the object surface;

(b) The voxel is not an end point;

(c) The deletion of the voxel would not change the Euler characteristics (i.e. creating new holes in the image);

(d) The deletion of the voxel would not change the number of connected components in the image.

In the skeleton representation, the segmented vessels are all represented by one-voxel wide centerlines, regardless of their widths. The centerlines preserve some key properties of the vessel network. Some properties, such as vessel lengths, branches and topological structures, can be easily computed on skeletons, though we still need the whole vascular network for the computation of some other properties (e.g., vessel thickness, which is a key property of vessels, is not reflected in the skeletons). Fig. 4(d) shows the skeleton of a 3-D vascular network.

\subsubsection{Error pruning}

The computed skeletons usually contain a large number of branches. However, most of the branches do not correspond to real structures. We identify the following two types of fake branches in the images.

(I) Most short vessel segments with only one end connected to long vessels are caused by non-smooth vessel surfaces. Most existing methods for pruning skeletons depend on a significance measure for each axis/centerline point. ${ }^{17}$ Extensions of the algorithm include axis propagation velocity, ${ }^{18}$ the $\lambda$-medial axis, ${ }^{19}$ and the discrete curve evolution. ${ }^{20}$ The significance score of each point is determined by its relative distance to the surface or outer medial axis. Voxels with low significance scores are pruned away. Kim et al. ${ }^{21}$ and Liu et al. ${ }^{22}$ gave a pruning algorithm to prune away false short branches in fibrin networks. The algorithm by $\mathrm{Kim}^{21}$ et al. and Liu et al. ${ }^{22}$ is based on the observation that most fake branches are caused by very small perturbations on the fiber surface, and small perturbations on the surface do not affect the outer medial axis much. False branches are identified based on their lengths and the distances from the branch points to the outer medial axes.

In our images, even after the pre-processing steps, the vessel surfaces could still be very unsmooth. In the computed skeletons, both real and fake branches are usually dense, and the fake branches could be as long as several voxels. Furthermore, fake branches may also contain multiple sub-branches. As a result, the distance from branch points to surfaces or out medial axes do not show obvious patterns.

We iteratively prune away such short vessel segments based on their lengths. Based on our experiment, the threshold value of 5 voxels can effectively remove most these fake short branches without affecting real vessel segments. Fig. 5(a) and 5(b) show part of a vascular network before and after pruning away short vessel segments, respectively.

(II) Fig. 5(c) and 5(d) show another possible cause for error that may occur when computing properties of vessel centerlines. Since the vessels are sometimes very dense, some vessels may seemingly touch each other in 3-D images. These vessels may or may not actually touch each other, but high-density concentrations of red dextran particles that leak outside of vessels may "connect" some vessels that are close to each other. These "touching" points will result in multiple branch points in the skeleton, but none of them should be considered as a real branch point.

In Fig. 5(d), the short vessel segment $\overline{u v}$ in the middle and its two branch points $u$ and $v$ are caused by two touching yet non-intersecting vessels as shown in Fig. 5(c). This false vessel segment, along with the two branch points that it connects, need to be removed from the skeleton. The general patterns of this type of false vessel segments are, for each of the two end points of the vessel segment, the two vessel branches from the same end point have approximately opposite orientations. In addition, the length of the vessel segment in the middle is close to the sum of the radii of the two vessels that it connects. In Fig. 5(d), vessel segments $a$ and $b$ have opposite orientations from the branch point, so do vessel segments $c$ and $d$.

We modify the algorithm by Chen et al. for identifying erroneously doubled branch points caused by intersecting fiber segments ${ }^{23}$ to deal with this type of error. The intersecting fiber segments in the paper by Chen et al. appear to be on the same plane, so do the computed fiber centerlines and the short segment in the middle. The orientation of the fiber segments, along with the size of fiber segments and length of the segment in the middle, are used for identifying erroneously doubled branch points. In our images, the vessels causing this type of error usually appear on different planes. Therefore, we modify the algorithm accordingly. 


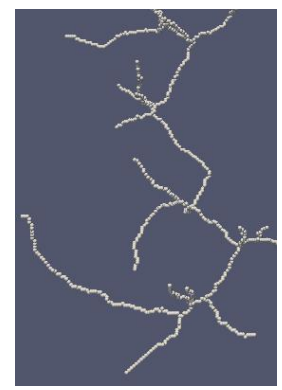

(a)

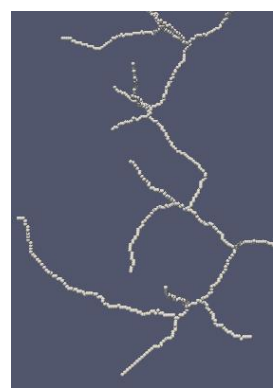

(b)

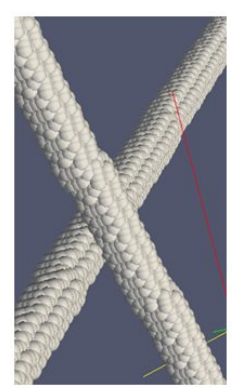

(c)

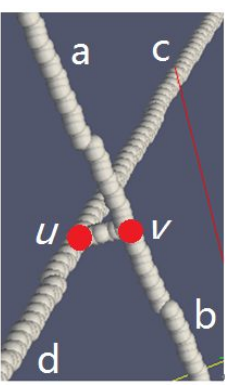

(d)

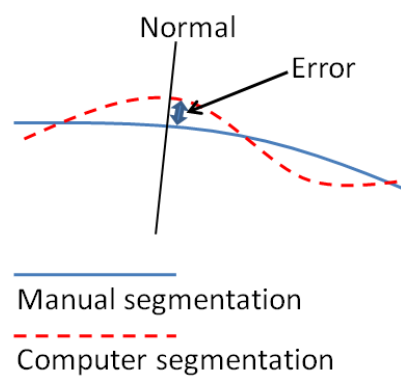

(e)

Figure 5. Post-processing and evaluation of vessel segmentation: (a) Part of a vascular network with fake short vessel segments; (b) the vascular network with the fake short vessel segments pruned away; (c) two vessels touching each other; (d) pruning fake branches caused by touching vessels; (e) evaluation of vessel segmentation.

We compute the orientations of the four vessel segments, $a, b, c$, and $d$, using linear regression. ${ }^{24}$ The purpose of linear regression is to fit a straight line through a sequence of sampled points on a curve to best represent the orientation of the curve. We take the following steps to compute the orientations of the vessel segments, and determine whether a vessel segment is fake.

(a) For each vessel segment (e.g., $\overline{u v}$ in Fig. $5(\mathrm{~d})$ ), we sample $n$ points $\left\{\left(x_{i}, y_{i}\right), i=1, \ldots, n\right\}$ from the four vessel segments (e.g., $a, b, c$ and $d$ in Fig. 5(d)) connected to the two branch points. In our algorithm, we sample 10 consecutive voxels from the branch point for each vessel segment connected to the branch points.

(b) We adopt the least-squares linear regression approach ${ }^{24}$ to compute the orientation for each vessel segment from the sampled voxels. The goal is to find a line that minimizes the sum of squared residuals (the vertical distances between the points of the data set and the fitted line), and can be written in the formula below:

$$
\text { Find } \min _{\alpha, \beta} Q(\alpha, \beta) \text {, for } Q(\alpha, \beta)=\sum_{i=1}^{n}\left(y_{i}-\alpha-\beta x_{i}\right)^{2},
$$

where $\alpha$ and $\beta$ are the $y$-intercept and slope of the computed line, respectively.

(c) We then compute the angles between the two pairs of vessel segments. We denote the angle between two directed vectors $p$ and $q$ as $\langle p, q\rangle$. In Fig. 5(d), if $\langle a, b\rangle>\Theta$ and $\langle d, d\rangle>\Theta$, where $\Theta$ is a predefined threshold, then we consider the vessel segment in the middle might be fake. Based on our experiment, we set the threshold value to $7 \pi / 8$.

(d) We measure the distances from the two end points of the vessel segment in the middle, to the nearest vessel surfaces, and use the shortest distances as the approximate vessel radii. In Fig. 5(d), we denote the shortest distances from $u$ and $v$ to their nearest vessel surfaces as $D_{u}$ and $D_{v}$, respectively, and use $D_{u}$ and $D_{v}$ as the approximate radii of the two vessels. If $\overline{u v}$ is fake, then $|u v|$ should close to $\operatorname{sum}\left(D_{u}, D_{v}\right)$. If $\overline{u v}$ is a fake vessel segment, $|u v|$ has to be in the range of $\left[0.8^{*} \operatorname{sum}\left(D_{u}, D_{v}\right), 1.2^{*} \operatorname{sum}\left(D_{u}, D_{v}\right)\right]$.

(e) If a vessel segment satisfies conditions in both (c) and (d), then we consider it as fake. The vessel segment is thus removed, along with the two branch points that it connects to.

\subsubsection{Quantitative Analysis}

We compute the following properties for the segmented vessels and hematopoietic cells.

1. Sizes of vascular networks. Vessels can be measured in their volume, length, and vessel segment thickness. The total volume can be measured by counting the number of voxels in the segmented vascular network. The total length can be measured by counting the number of voxels on the skeleton. The vessel segment thickness may be measured by the diameter or area of the vessel segment cross-section.

2. Vessel branching. Vessels often divide into two smaller vessels that go in different directions, like the branches of a tree. Branching is an important aspect of network topology. Branching patterns may affect the 
Table 1. Quantitative property comparison of the two image types.

\begin{tabular}{|c|c|c|c|c|c|c|c|c|}
\hline & $(\mathrm{a})$ & $(\mathrm{b})$ & $(\mathrm{c})$ & $(\mathrm{d})$ & $(\mathrm{e})$ & $(\mathrm{f})$ & $(\mathrm{g})$ & $(\mathrm{h})$ \\
\hline Controlled & 5977 & 102 & $1.71 \%$ & 26.99 & $3.75 \times 10^{6}$ & 629 & 894 & 8.50 \\
\hline Knock-out & 4045 & 77 & $1.90 \%$ & 27.02 & $3.82 \times 10^{6}$ & 952 & 1921 & 13.64 \\
\hline p-value & 0.08 & $0.05^{*}$ & 0.19 & 0.33 & 0.22 & $0.03^{*}$ & $0.01^{*}$ & $0.02^{*}$ \\
\hline
\end{tabular}

Computed features: (a) Total vessel length; (b) total number of branch points; (c) percentage of branch points; (d) average length of vessel segments; (e) total vessel volume; (f) average area of vessel cross-section; (g) cell count; (h) average distance from cells to the nearest vessels (* denotes properties with significant difference).

interaction between vessels and cells. Here we study properties of branch points of the vascular network and vessel segments separated by branch points, which reflect the branching patterns. The more branch points there are in the network, the more bifurcations the vessels have. We count the total number of branch points, and compute the percentage of branch points among all voxels on the skeleton. We also compute the average length of all vessel segments separated by branch points.

3. Cell count and distribution. We count the total number of segmented cells, and compute the average distance from each cell to the nearest vessel surface. This distance may reflect the degree of interaction between cells and vessels.

We analyze 19 controlled and 16 knock-out type images. The controlled samples are from normal mice and the knock-out samples are from mice with Notch signaling pathway deleted to simulate diseases related to Notch signaling pathway (i.e., dysfunction or disorder of Notch pathway). Table 1 shows the comparison of the computed features between the two image types. Statistically significant differences are observed in properties (b), (f), (g), and (h). Comparing with the controlled samples, the knock-out samples tend to have: (1) fewer branch points (although the branch point density is roughly the same); (2) thicker vessels; (3) more cells surrounding the vessels, but the cells are farther away from the vessel surfaces.

Our analysis results show that, with impaired regulation from Notch signaling pathway, the development of vascular network is less controlled. The vessels expand in size, and this expansion engulfs some small vessels, resulting in the decrease in the number of branch points and total vessel length (although the difference shown in the total vessel length is not considered statistically significant as indicated by the $p$-value). Meanwhile, the bone marrow microenvironment lost some hematopoietic cell. However, more hematopoietic cells are generated to engage in the recovery process of bone marrow microenvironment. This explains why more hematopoietic cells were observed but the cells are farther away from vessel surfaces compared with controlled samples.

Our analysis quantitatively illustrates how deleted Notch pathway affects the bone marrow microenvironment, and how the vascular structure and hematopoietic cell distribution changed in the bone marrow. Our algorithms can be used to quantitatively measure changes in the bone marrow microenvironment caused by various diseases or factors, and monitor the recovery during treatment.

\section{EVALUATION}

We manually segment the vessel surfaces in 3-D. The accuracy of vessel segmentation is evaluated by measuring the average distance between the computer-segmented and manually-segmented vessel surfaces. As shown in Fig. 5(e), points are sampled on the surface of the manually-segmented surface, and the normal at each sampled point is drawn. The normal intersects both the manually-segmented (the solid curve) and computer-segmented surfaces (the dashed curve), the gap between the two surfaces is the error at this point. We measure both signed and unsigned errors, and report the errors in mean \pm standard deviation.

The cells and branch points are manually labeled in 3-D, and compared with computer-generated results. False positive (FP) and false negative (FN) rates are reported. In some images, there are some highly fuzzy regions which may contain red or green voxels of high density, but no vascular or cellular structures with clear boundaries can be seen. Although our algorithm may still identify some vessels or cells in these regions, we exclude these regions from the evaluation. 
Table 2. Evaluation of vessel and cell segmentation.

\begin{tabular}{|l|c|c|c|c|c|c|}
\hline & \multicolumn{2}{|c|}{ Vessel Segmentation } & \multicolumn{2}{c|}{ Cell Segmentation } & \multicolumn{3}{c|}{ Branching } \\
\hline & signed err. (voxels) & unsigned err. (voxels) & FP (\%) & FN (\%) & FP (\%) & FN (\%) \\
\hline Controlled & $2.09 \pm 0.56$ & $4.95 \pm 1.33$ & 6.67 & 5.62 & 8.85 & 5.16 \\
\hline Knock-out & $2.64 \pm 0.58$ & $6.37 \pm 1.71$ & 9.96 & 7.56 & 13.17 & 5.56 \\
\hline
\end{tabular}

Evaluation is performed to assess the accuracy of vessel segmentation, cell segmentation and branch point identification. Signed/unsigned errors, false positive (FP) and false negative (FN) rates are computed.

We randomly selected 3 controlled and 3 knock-out type 3-D images for evaluation. Table 2 shows the evaluation results. In general, the results on knock-out samples are less accurate than on controlled samples, since the images for knock-out samples are more fuzzy and contain more imaging artifacts.

\section{CONCLUSIONS}

We present and evaluate algorithms that can effectively segment vascular structures and hematopoietic cells in 3-D microscopy images, and perform quantitatively analysis of their properties. We find that after the Notch signaling pathway is deleted, the vessels in the bone marrow become thicker and the number of branch points is reduced. Meanwhile, more hematopoietic cells are found surrounding the vessels, but the cells are farther away from the vessel surfaces compared with controlled samples. Our findings help biologists study how the dysfunction of Notch signaling pathway affects and bone marrow microenvironment, monitor the recovery process and develop therapeutic strategies. The platform we develop is a useful tool for biologists to study changes in the bone marrow microenvironment caused by various diseases or other factors.

\section{ACKNOWLEDGEMENTS}

This work was supported in part by NSF under grant CCF-0515203 and the Indiana Clinical and Translational Sciences Institute (ICTSI) under NIH/NCRR Grant UL1TR001108.

\section{REFERENCES}

[1] Allman, D., Aster, J. C., and Pear, W. S., "Notch signaling in hematopoiesis and early lymphocyte development," Immunological Reviews 187(1), 75-86 (2002).

[2] Bigas, A. and Espinosa, L., "Hematopoietic stem cells: to be or Notch to be," Blood 119(14), 3226-3235 (2012).

[3] Ribatti, D., "Bone marrow vascular niche and the control of tumor growth in hematological malignancies," Leukemia 24(7), 1247-1248 (2010).

[4] Wang, L., Zhang, H., Rodriguez, S., Cao, L., Parish, J., Mumaw, C., Zollman, A. L., Kamocka, M. M., Mu, J., Chen, D. Z., Srour, E. F., Chitteti, B. R., HogenEsch, H., Tu, X., Bellido, T. M., Boswell, H. S., Manshouri, T., Verstovsek, S., Yoder, M. C., Kapur, R., Cardoso, A. A., and Carlesso, N., "Notch-dependent repression of miR-155 in the bone marrow niche regulates hematopoiesis in an NF-kB-dependent manner," Cell Stem Cell 15(1), 51-65 (2014).

[5] Lam, B. S. Y. and Yan, H., "A novel vessel segmentation algorithm for pathological retina images based on the divergence of vector fields," IEEE Transactions on Medical Imaging 27(2), 237-246 (2008).

[6] Marin, D., Aquino, A., Gegundez-Arias, M. E., and Bravo, J. M., "A new supervised method for blood vessel segmentation in retinal images by using gray-level and moment invariants-based features," IEEE Transactions on Medical Imaging 30(1), 146-158 (2011).

[7] Descombes, X., Plourabou, F., Boustani, A., Fonta, C., Duc, G. L., Serduc, R., and Weitkamp, T., "Vascular network segmentation: An unsupervised approach," in [Proceedings of the 9th IEEE International Symposium on Biomedical Imaging], 1248-1251 (2012).

[8] Vlachos, M. and Dermatas, E., "Multi-scale retinal vessel segmentation using line tracking," Computerized Medical Imaging and Graphics 34(3), 213-227 (2009). 
[9] Staal, J., Abramoff, M. D., Viergever, M. X., and Ginneken, B. V., "Ridge-based vessel segmentation in color images of the retina," IEEE Transactions on Medical Imaging 25(4), 501-509 (2004).

[10] Soares, J. V. B., Leandro, J. J. G., Cesar, R. M., Jelinek, H. F., and Cree, M. J., "Retinal vessel segmentation using the 2-D gabor wavelet and supervised classification," IEEE Transactions on Medical Imaging 25(9), 1214-1222 (2006).

[11] Rouchdy, Y. and Cohen, L. D., "Retinal blood vessel segmentation using geodesic voting methods," in [Proceedings of the 9th IEEE International Symposium on Biomedical Imaging], 744-747 (2012).

[12] Dufour, A., Passat, N., Naegel, B., and Baruthio, J., "Interactive 3D brain vessel segmentation from an example," in [Proceedings of the IEEE International Symposium on Biomedical Imaging: From Nano to Macro], 1121-1124 (2011).

[13] Babin, D., Pizurica, A., Vylder, J. D., Vansteenkiste, E., and Philips, W., "Brain blood vessel segmentation using line-shaped profiles," Physics in Medicine and Biology 58(22), 8041-8061 (2013).

[14] Tang, C. and Bengtsson, E., "Segmentation and tracking of neural stem cell," Advances in Intelligent Computing, Lecture Notes in Computer Science 3645, 851-859 (2005).

[15] Wait, E., Winter, M., Bjornsson, C., Kokovay, E., Wang, Y., Goderie, S., Temple, S., and Cohen, A. R., "Visualization and correction of automated segmentation, tracking and lineaging from 5-D stem cell image sequences," BMC Bioinformatics 15(328) (2014).

[16] Lee, T. C., Kashyap, R. L., and Chu, C. N., "Building skeleton models via 3-D medial surface axis thinning algorithms," Computer Vision, Graphics, and Image Processing 56(6), 462-478 (1994).

[17] Shaked, D. and Bruckstein, A., "Pruning medial axes," Computer Vision and Image Understanding 69(2), 156-169 (1998).

[18] Leymarie, F. and Levine, M. D., "Pruning medial axes," IEEE Transactions on Pattern Analysis and Machine Intelligence 14(1), 56-75 (1992).

[19] Chazal, F. and Lieutier, A., "The $\lambda$-medial axis," Journal of Graphical Models 67(4), 304-331 (2005).

[20] Bai, X., Latecki, L. J., and Liu, W. Y., "Skeleton pruning by contour partitioning with discrete curve evolution," IEEE Transactions on Pattern Analysis and Machine Intelligence 29(3), 449-462 (2007).

[21] Kim, E., Kim, O. V., Machlus, K. R., Liu, X., Kupaev, T., Lioi, J., Wolberg, A. S., Chen, D. Z., Rosen, E. D., Xu, Z., and Alber, M. S., "Correlation between fibrin network structure and mechanical properties: An experimental and computational analysis," Soft Matter 7(10), 4983-4992 (2011).

[22] Liu, X., Mu, J., Machlus, K. R., Wolberg, A. S., Rosen, E. D., Xu, Z., Alber, M., and Chen, D., "Automatic segmentation and analysis of fibrin networks in 3D confocal microscopy images," in [Proceedings of the SPIE International Symposium on Medical Imaging: Image Processing], 8314 (2012).

[23] Chen, J., Kim, O. V., Litvinov, R. I., Weisel, J. W., Alber, M. S., and Chen, D. Z., "An automated approach for fibrin network segmentation and structure identification in 3D confocal microscopy images," in [Proceedings of the 27th IEEE International Symposium on Computer-Based Medical Systems], 173-178 (2014).

[24] Seber, G. A. F. and Lee, A. J., [Linear Regression Analysis], John Wiley \& Sons (2012). 